\title{
Physical tests in the selection of electrical lineman depending on the cardiac rhythm when working at height for the first time
}

\author{
Ernesto A. Abril ${ }^{1}$, Ernesto M. Abril ${ }^{1}$, Diana D. Marín ${ }^{1}$, Leonardo M. Abril ${ }^{2}$ \\ ${ }^{1}$ Facultad de Ciencias de la Ingeniería y Aplicadas, Universidad Técnica de Cotopaxi , Latacunga-Ecuador \\ Departamento de Ciencias Económicas Administrativas y de Comercio \\ ${ }^{2}$ Universidad de las Fuerzas Armadas ESPE, Sangolquí-Ecuador
}

\begin{abstract}
In the present work of investigation, the pulse cardiac is monitoring with a heart rate sensor. All data record is obtained of a healthy person group, five junior and one expert electric lineman, Pulse cardiac let show the behavior of the heart when responding to physical effort and stress situation. So that we relate the required physical effort evidenced by a calorific map, the condition of stress due to the fear of falling and the variability of the cardiac pulse. Study is presented in stages. In the first stage, it is possible to demonstrate the increase of the cardiac pulse due to the effort. In the second stage, the increase of the cardiac pulse due to a condition of stress or fear is presented. Finally, in the third stage, the cardiac pulse increases proportionally. Also the cardiac pulse is monitored when descending the concrete post. A variability of the cardiac rhythm between the participants is obtained. It checks that the heart rate after an effort or stress condition becomes unpredictable for different people.
\end{abstract}

\section{Introduction}

In the electrical sector, the cardiological effects produced by work on electrical towers and concrete poles have not been studied. The heart rate in the electrical linemen allows to determine if a danger will occur due to its nervous state. This is because when working in medium voltage, an error can make the difference between life and death.

On the other hand, the cardiological information of the prospects to begin in this high-risk work is of vital importance. This is because the electric lineman, when presenting a state of anxiety, would cause him to make an error in the medium voltage lines. Anxiety is clearly observed in cardiac pulse curves with high pulse-to-pulse variability. Showing steep slopes.

The electric lineman is in charge of maintaining the high voltage towers and the distribution lines. Infrastructure that allows the transport of energy from generators to consumers. Manuals for the selection of personnel in the electrical line work post indicate that it is necessary to monitor the cardiac pulse of the prospects. The pulse must be kept below the maximum permissible. [1]

In the studies the physical and psychological behavior are presented. The recommendations are presented before, during and after performing the physical tests. This is done to get the best results.

For the physical tests is recommended, that each of the participants avoid the consumption of tobacco and perform aerobic and walking activities. [1]
Cardiac pulse monitoring is essential to analyze the behavior of the heart when performing physical activities and stress situations. Most cardiological studies require the acquisition and analysis of heart signals. For this reason, heart rate sensors and ergometers are used.

This monitoring of cardiac pulse has served to validate physical activity questionnaires. Since it allowed to demonstrate significant correlations between the cardiac pulse and physical activities in different levels of intensity. The study method consisted of analyzing a multi-ethnic sample from New Zealand, which consisted of people from the Pacific, Maori and Europeans.

The multi ethnic people were monitored during a period of three consecutive days, to later associate the cardiac pulse to the different activities of a daily day. Ultimately the study aimed to establish a guide with suggestions of physical activities to maintain a good state of health in the local population.

On the other hand, the statistical analysis is based on the recommendations by the American College of Sports Medicine, which establishes that for moderate exercises there will be a cardiac pulse reserve of $40 \%$ to $50 \%$ and for high intensity exercises of $60-70 \%$ and the maximum cardiac pulse is:

$\mathrm{HR}_{\text {max }}=220$-age

In which $\mathrm{HR}_{\max }$ is the maximum cardiac pulse depending on the age of the person. [2]

The cardiac pulse has been studied for many years. It is because there are many devices to analyze these signals such as electrocardiograms. The device records the frequency and variability of the cardiac pulse. 
In the psychological aspect the heart rate as a measure of stress or anxiety. It has been noted that depending on the state of mind and under extreme conditions a person can increase or decrease their heart rate. However, this variability cannot be greater than unity.

Experiments were performed in which the heart rate was checked before, during (exposure of the individual to a stress condition) and afterwards. This condition consisted in keeping one foot on ice. From the experiment it was observed that in a stress condition the person accelerates his heart rate.

In another experiment, an individual with good physical condition was observed. It consisted in that he performs push-ups to the point of exhaustion, then the data obtained of cardiac pulse in the post-stress stage are unstable and unpredictable. [3]

In other experiment shows that the pulse and heart rate varies, depending on whether the person is asleep or awake. Four people were employed in good health. These people would make a trip of 5 hours. During the trip they had to press a button in case of feeling drowsy. Throughout the experiment people were being monitored.

The data extracted indicated a $10-20 \%$ decrease in the cardiac pulse and a $10 \%$ increase in the variability of the cardiac pulse. [4]

The cardiac pulse has also been linked to phobias. In some experiments people with spider phobia have been used. In the subjects, the cardiac pulse was monitored before and after some tests. The tests consisted of touching a spider's web to touching a living spider. The data from the experiment show an increase in the cardiac pulse when the subjects face a phobia. [5]

Experiments have been developed that involve people in good physical condition. The people were subjected to continuous and constant bicycle exercises. The cardiac pulse was analyzed as a function of time and presented two components. [6] The first component presented an abrupt increase and in the second component the cardiac pulse stabilized with a cardiac pulse close to the maximum HRmax. [2] [6]

The results were obtained in the city of Ambato, Ecuador in South America. The city is located at a height of 2700 meters above sea level. The electric distribution lines expand to areas far from the cities, for this reason the work sectors are above 3000 meters above sea level. These working conditions require a greater physical effort and therefore a greater number of cardiac pulses by the electric line.

Electrical linemen are equipped with foot couplings, seat belts and helmets. The foot couplings are hooked on the concrete poles in each step that is given to the ascent. The weight of the equipment is 30 [kg]. Before the start of the physical tests, the participants were weighed, if their weight exceeded 285 pounds they were not taken into account for the tests. Smoking and eating were not allowed in the course of the tests, as was the use of cell phones. [7]

The study presents two parts of observation in the first one show the caloric corporal points in which greater effort is made, in the second part the curves of the cardiac pulse of each one of the participants are obtained. Finally the two parts of the experiment are analyzed.

\section{Development}

\subsection{Electrical lineman thermal body map}

Initially, the body thermal map was observed. An increase in temperature is observed in the areas in which greater effort is required Figure 1 . The progress of the electric lineman in the ascent to the pole is observed.

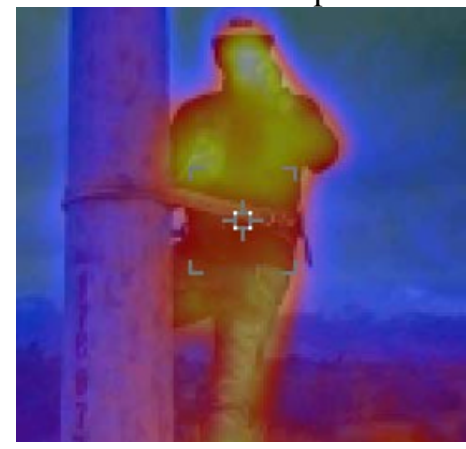

Figure 1. Start climbing

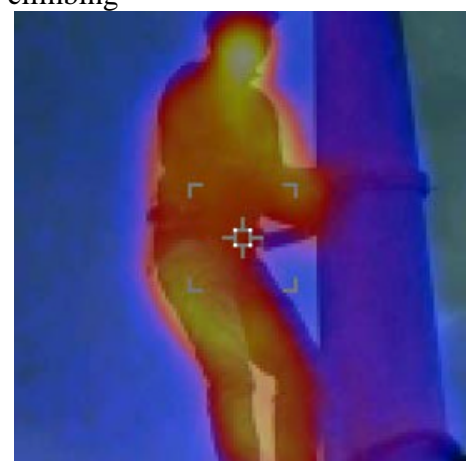

Figure 2. Middle climbing

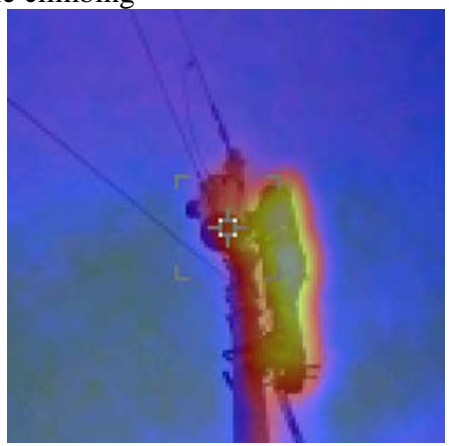

Figure 3. Finish climbing

The images show a concentration of heat in the arms, chest and legs. Figure 2. Thermal radiation protrudes above the clothes. The body radiation emitted by the face of the person is discarded.

At the end of the ascent, the highest concentration of heat is found in the thighs. This is due to the greater effort required. Figure 3.

\subsection{Cardiac pulse monitoring rise.}

The heart rate was recorded using a sensor with electrodes. The sensor has communication by bluetooh to record the data. 
Six people participated in the experiment. A person has experience in this activity. The other 5 people carry out this activity for the first time.

The cardiac pulse curves of Figures 1, 2, 3, 4, 5and 6 are from junior linemen as they ascend.

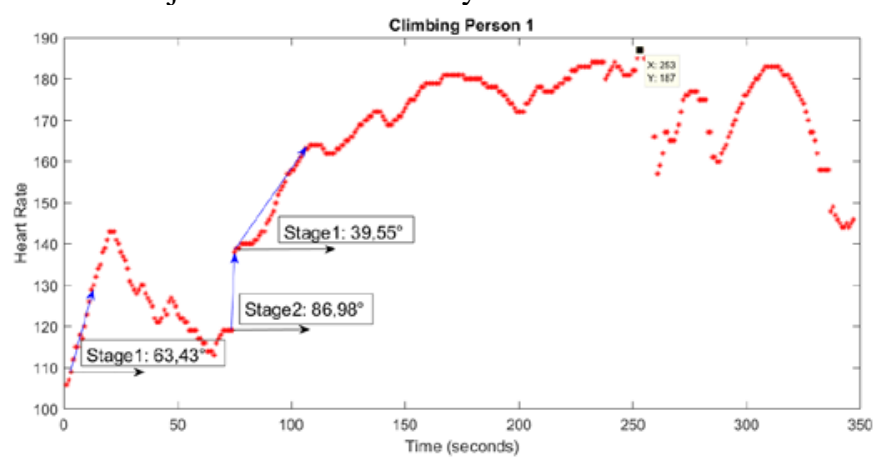

Figure 4. Person 1 Climbing by stages.

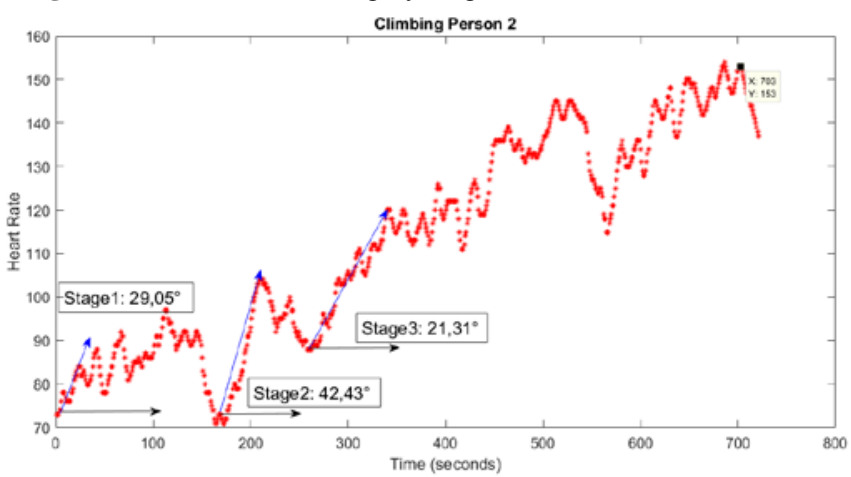

Figure 5. Person 2 Climbing by stages.

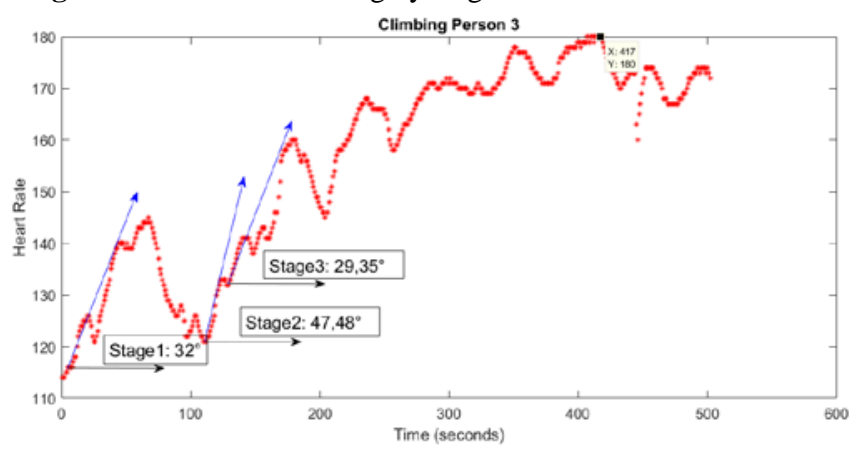

Figure 6. Person 3 Climbing by stages

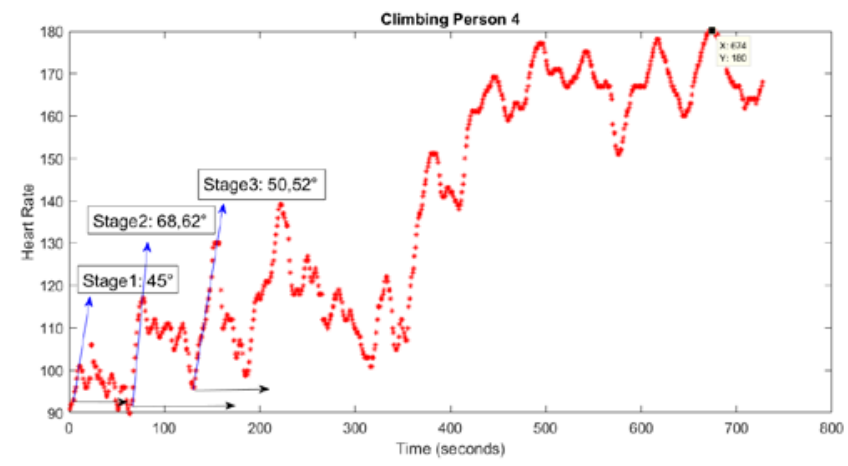

Figure 7. Person 4 Climbing by stages

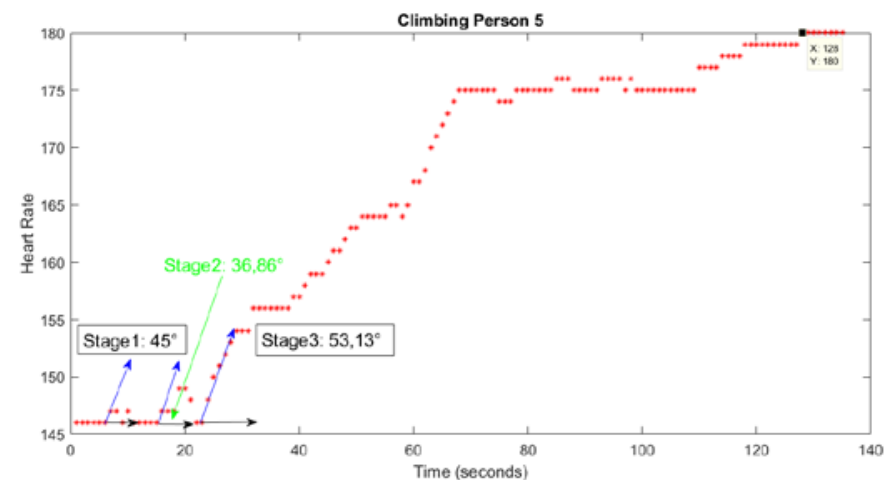

Figure 8. Person 5 Climbing by stages

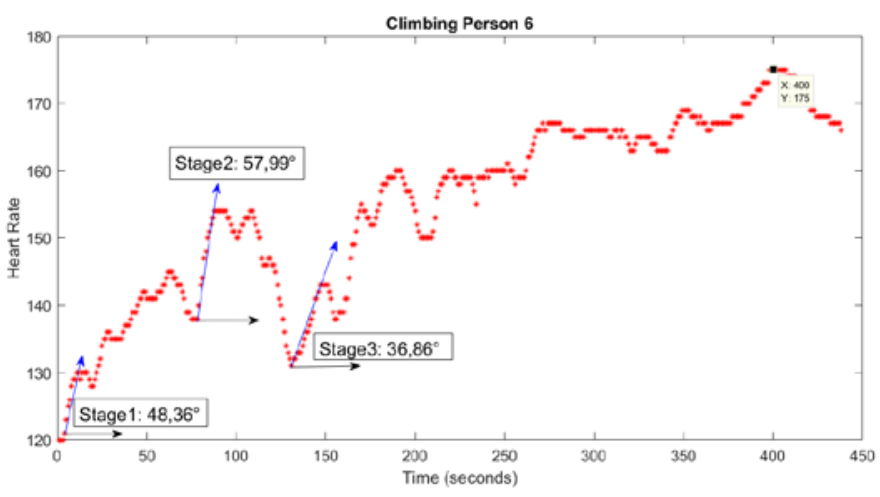

Figure 9. Person 6 Climbing by stages

\subsection{Cardiac pulse monitoring when descending.}

The maximum heart rate [1] was fixed according to the average age of the group of people observed. The average age is 30 years.

$\mathrm{HR}_{\text {max }}=220$-age

$$
\mathrm{HR}_{\max }=220-30=190
$$

At the end of the climb and start the descent the following curves are presented. The blue line is the maximum cardiac frequency that is permissible. Figure $10,11,12,13,14$ and 15.

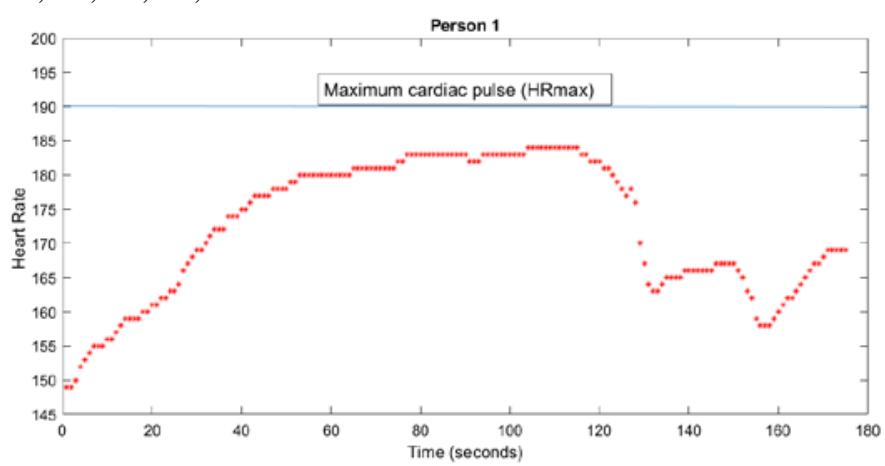

Figure 10. Person 1 Descending 


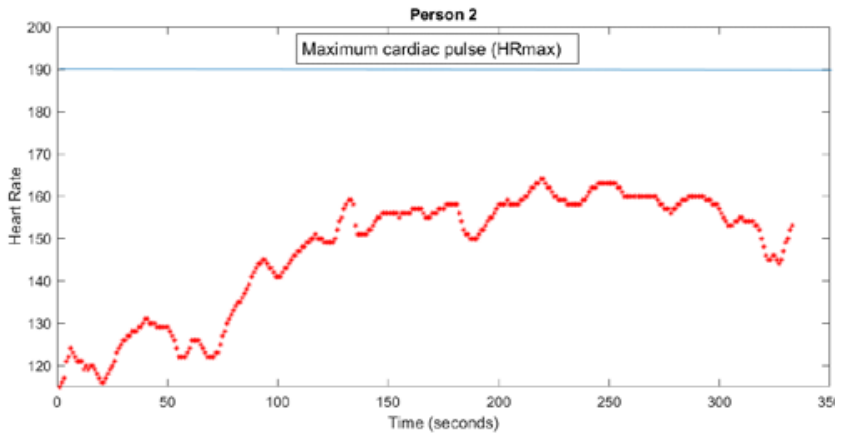

Figure 11. Person 2 Descending

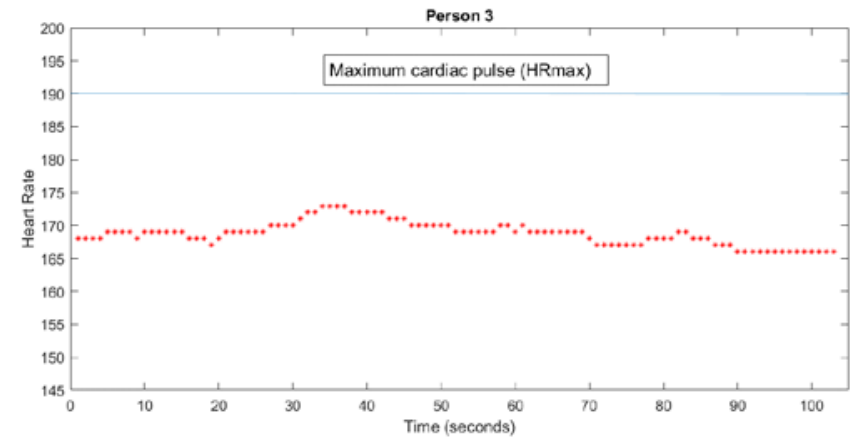

Figure 12. Person 3 Descending

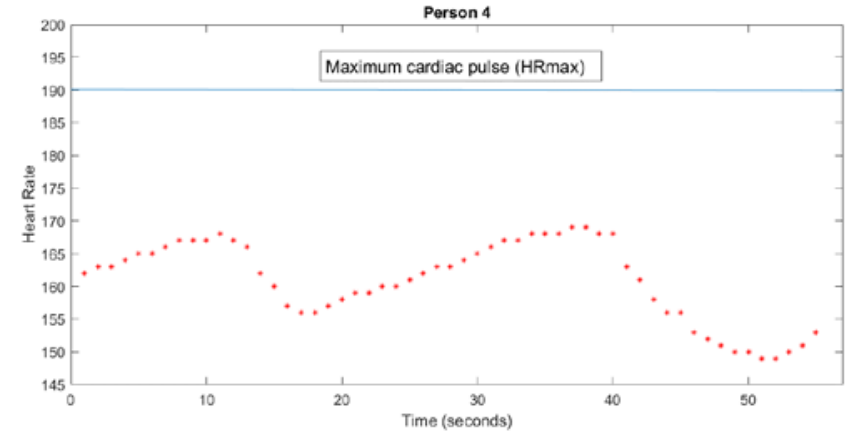

Figure 13. Person 4 Descending

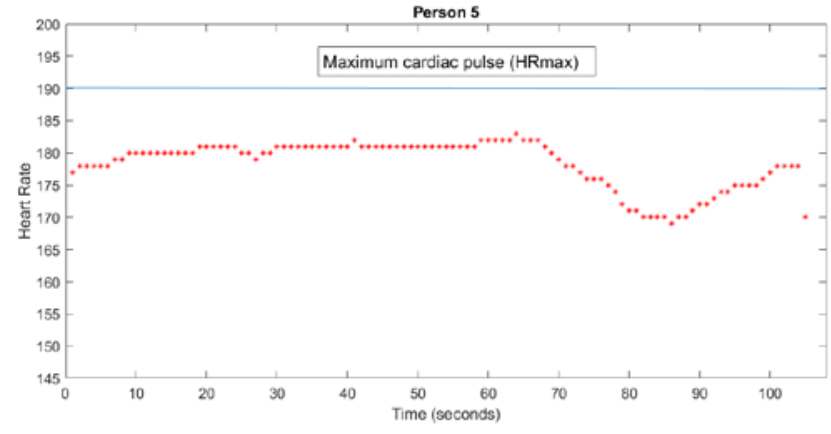

Figure 14. Person 5 Descending

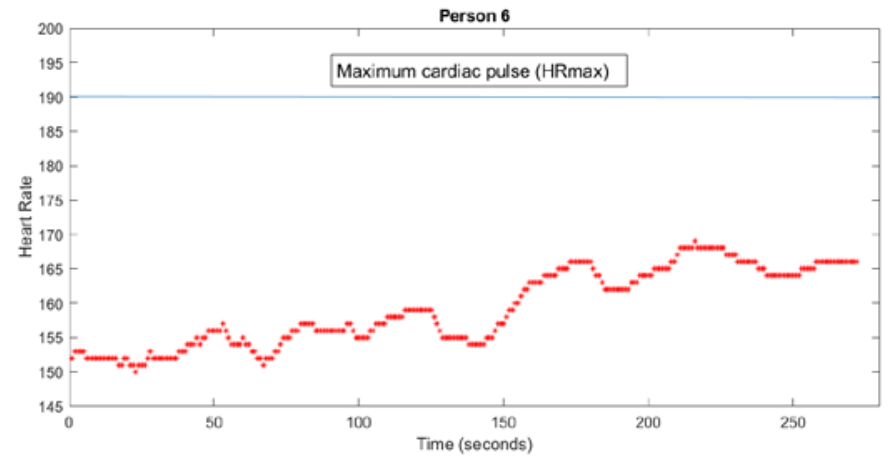

Figure 15. Person 6 Descending

\section{ANALYSIS OF RESULTS}

According to each of 6 images the column of stage 1 present the first step, the stage 2 is the second step and stage 3 represent steps for climbing all concrete post. Table 1.

In stage 1 it gives the first step on the concrete post but still keeps the other foot on the ground. This is what reflects the effort that this first step requires. In stage 2, he gives the second step on the concrete post. The person loses contact with the ground and for this reason is in a state of anxiety. Due to the fear of falling. In stage 3, the vertical ascent to the concrete post begins. For this reason every step that the union of fear and effort presents, maintaining a constant and continuous increase up to the limit of the cardiac frequency.

Table 1. Climbing with data angular slope by stages

\begin{tabular}{|l|c|c|c|}
\cline { 2 - 4 } \multicolumn{1}{c|}{} & \multicolumn{3}{c|}{ Angular slope climbing } \\
\cline { 2 - 4 } \multicolumn{1}{c|}{} & Stage 1 & Stage 2 & Stage 3 \\
\hline Person 1 & $63.43^{\circ}$ & $86,98^{\circ}$ & $39,55^{\circ}$ \\
\hline Person 2 & $29,05^{\circ}$ & $42,43^{\circ}$ & $21,31^{\circ}$ \\
\hline Person 3 & $32^{\circ}$ & $47,48^{\circ}$ & $29,35^{\circ}$ \\
\hline Person 4 & $45^{\circ}$ & $68,62^{\circ}$ & $50,52^{\circ}$ \\
\hline Person 5 & $45^{\circ}$ & $36,86^{\circ}$ & $53,13^{\circ}$ \\
\hline Person 6 & $48,36^{\circ}$ & $57,99^{\circ}$ & $36,86^{\circ}$ \\
\hline
\end{tabular}

Results of Table 1, Of the 6 people monitored, the fifth one has experience in this type of work. This is evidenced by the lower cardiac pulse in the second stage compared to the other participants. The rest of the participants carry out this activity for the first time.

The inexperienced participants have a greater heart rate in the second step they take when climbing the concrete post. This is due to the fear of falling since from there they fall off the ground.

However, in the third stage, the increase of the cardiac pulse in both the experienced participant and those without experience, maintain a continuous and constant increase..

When starting with the descent of the concrete post, the cardiac pulse in each one of the participants becomes unpredictable and variable. 


\section{Conclusions}

The cardiac pulse tends to increase when a person is in a state of stress and stress. The heart rate allows to differentiate each one of these states. Coinciding with greater variability in the second stage effect of the state of stress and fear. However, in people with experience in this type of work the heart rate decreased with respect to the first stage, denoting the absence of the effects of stress. When concluding with the ascent, the cardiac rhythm is difficult to predict. This is because the physical state and physiology of each of the participants is different. Causing different forms of curve in each of them.

\section{Acknowledgment}

Our sincere thanks to Universidad Técnica de Cotopaxi for collaborating with the present project with the logistics for the respective presentation and Msc. Idalia Pacheco for the eloquent administrative management.

\section{References}

1. Pacific Gas and Electric Company, "Post-Offer Physical Assessment - Lineman Line of Progression”, PG\&E Corporation, Available: www.pge.com [Accessed Febrary. 14, 2017]
2. Karen L. Moy, Robert K. Scragg, Grant McLean, and Harriette Carr, "The New Zealand Physical Activity Questionnaires: Validation by Heart-Rate Monitoring in a Multiethnic Population”, Journal, Physical Activity \& Health vol 5, pp. 45-61, 2008.

3. J. Alan Burdick and John T. Scarbrough, "Heart Rate and Heart Rate Variability an Attempt to Clarify”, Journal, Perceptual and Motor Skills vol 26, pp. 1047-1053, 1968.

4. Sang-Ho Jo, Jinmyung Kim and Dongkyu Kim, "Heart Rate and Heart Rate Variability Change While Falling Asleep During Driving a Car”, Journal, American College of Cardiology vol 70, pp. 79, 2017.

5. C. B. Taylor, "Heart-Rate Changes in Improved Spider-Phobic Patients”, Journal, Psychological Reports, vol 41, pp. 667-671, 1977.

6. W. Michael Felts, Stephen Crouse and Mark Brunetz "Influence of Aerobic Fitness on Ratings of Perceived Exertion during Light to Moderate Exercise”, Journal, Perceptual and Motor Skills , pp. 671-676, 1988.

7. Ediscon International Company, "Lineman Physical Performance Test Battery”, Southern California Edison, Available: www.edison.com [Accessed Febrary. 15, 2017] 